BULL. AUSTRAL. MATH. SOC.

VOL. 27 (1983), 347-360.

\title{
ON DECOUPLING OF LINEAR RECURSIONS
}

\author{
R.M.M. Mattheis
}

\begin{abstract}
We show how a well known algorithm to compute solutions of a second order recursion which are unstable both in forward and in backward direction, can be related to a number of other methods. They are: order reduction, invariant imbedding and decoupling based on triangularization. It is shown that these methods in this order form an increasingly general approach to solve the problem. In particular this means that the stability of the first three algorithms can be understood from the theory that has been established for the decoupling algorithm. In this way one does not need to investigate the stability of the large sparse system which is often related to the first method.
\end{abstract}

\section{Introduction}

In 1967 Olver [10] published a method for computing a subdominant solution to a second order scalar recursion. Since then many modifications, adaptations and generalizations have been discussed in the literature; see, for example, Cash [1, 2], Lozier [3], Olver [9], Olver and Sookne [11]. The basic idea of the method, as it was described in Olver [10] was inspired by the fact that the recurrence relations, the initial condition and the dominance requirement together give rise to an infinite dimensional system of linear equations. By replacing the condition at infinity by one at say $N$, one obtains a finite linear system which, in principle, can be solved by $L U$-decomposition. Since then a

Received 25 January 1983. This work was in part supported by grants of the Netherlands Organization for the Advancement of Pure Research (ZWO) and the Niels Stensen Stichting. 
number of variants have appeared which were based on similar considerations for solving this large sparse system. Although the approach of Olver [10] virtually eliminates the problem of providing a terminal condition for the solution, there still remains the problem that the large linear system does not lend itself to a simple stability analysis. Indeed, a standard way to do this is to consider the effect of small perturbations of the matrix coefficients on the solution vector, see Wilkinson [12]. This gives, at its best, norm estimates for the global perturbation vector, but does not say anything about relative errors in the components (which form the desired solution of the recursion): nevertheless, the often exponential character of the solution necessitates the latter type of analysis. Also, it is not clear from scratch why such a matrix should be well-conditioned and in particular no estimates of its inverse are available. In this note we show that one does not need the large linear system approach. Indeed, it will turn out in the sequel that the algorithm described in 0lver [10] may as well be interpreted as a special case of order reduction (Section 3), the Riccati method or invariant imbedding (Section 4) and finally decoupling based on triangularization (Section 5). In this order those algorithms turn out to form an increasingly general approach to compute subdominant solutions of linear recursions.

In particular, by interpreting these algorithms as a decoupling method one can apply the fairly general stability analysis of Mattheij $[4,5,6]$. As an example this is carried out for the Riccati method.

\section{0lver's algorithm}

We briefly describe Olver's algorithm in this section. Assume we have the recurrence relation for the solution $\left\{x_{i}\right\}_{i \geq 0}$ :

$$
x_{i+1}=a_{i} x_{i}+b_{i} x_{i-1}+d_{i}, i=1,2, \ldots,
$$

where the initial value of this solution is given by

$$
x_{0}=c
$$

The algorithm then first computes a solution $\left\{y_{i}\right\}_{i=0}^{N+1}$ (the value of $N$ is not discussed here) of the homogeneous recursion 


$$
y_{i+1}=a_{i} y_{i}+b_{i} y_{i-1}, i=1,2, \ldots, N \text {, }
$$

which satisfies the initial values

$$
y_{0}=0, y_{1}=1
$$

Then a solution $\left\{z_{i}\right\}_{i=0}^{N}$ of the recursion (which uses known $y_{i}$ values)

$$
z_{i}=-b_{i} z_{i-1}-d_{i} y_{i}, i=1,2, \ldots, N \text {, }
$$

is computed, using the initial value

$$
\boldsymbol{z}_{0}=c
$$

It then follows that the desired solution $\left\{x_{i}\right\}_{i=0}^{N+1}$ of (2.1) must satisfy the relation

$$
z_{i}=y_{i+1} x_{i}-y_{i} x_{i+1}
$$

Since $\left\{x_{i}\right\}_{i \geq 1}$ is unknown, one may now compute an approximate sequence say $\left\{x_{i}^{(N)}\right\}_{i=0}^{N+1}$, by defining

$$
x_{N+1}^{(N)}=0
$$

and $(c f .(2.7))$

$$
x_{i}^{(N)}=\left[z_{i}+y_{i} x_{i+1}^{(N)}\right] / y_{i+1}, i=N, N-1, \ldots, 1,
$$

(where we assume, for all $i, y_{i} \neq 0$ ).

In Olver [10] it has been shown that under fairly general conditions $x_{i}^{(N)} \rightarrow x_{i}$ for $i$ fixed and $N \rightarrow \infty$, and that the recursions $(2.3),(2.5)$ and (2.9) are apparently stable in the indicated direction.

\section{Order reduction}

The second method we are going to discuss is perhaps the most obsolete one. If a certain solution to a linear scalar $n$th order homogeneous recursion is known, one can find an $(n-1)$ th order recursion that is 
satisfied by suitably transformed solutions of the original recursion; in particular, the reducing solution is a trivial solution of the latter recursion. This general so called order reduction technique can be found in standard textbooks, for example, [8]. Below we describe a generalized version for the inhomogeneous recursion (2.1).

Let a solution $\left\{\tilde{y}_{i}\right\}_{i=0}^{N+1}$ to the homogeneous recursion (2.3) satisfy the initial conditions

$$
\tilde{y}_{0}=A, \quad \tilde{y}_{1}=B ; A, B \in \mathbb{R} .
$$

Let $\left\{s_{i}\right\}_{i=0}^{N}$ be such that

$$
x_{i}=s_{i} \tilde{y}_{i}, i=0,1, \ldots, N \text {. }
$$

Then we obtain, from (2.1) and (3.2),

$$
\begin{aligned}
s_{i+1} \tilde{y}_{i+1}=s_{i} \tilde{y}_{i+1}+\left(s_{i-s_{i-1}}\right)\left(-\tilde{y}_{i+1}\right. & \left.+a_{i} \tilde{y}_{i}\right) \\
& +s_{i-1}\left(-\tilde{y}_{i+1}+a_{i} \tilde{y}_{i}+b_{i} \tilde{y}_{i-1}\right)+d_{i} .
\end{aligned}
$$

Defining the weighted differences

$$
t_{i}:=\left(s_{i+1} s_{i}\right) p_{i}, i=0,1, \ldots, N \text {, }
$$

where the $p_{i}$ are some nonzero real numbers, and noting that $\left\{\tilde{y}_{i}\right\}_{i=0}^{N+1}$ satisfies (2.3) we can reduce (3.3) to a first order recursion for $\left\{t_{i}\right\}_{i=0}^{N}:$

$$
t_{i}=-b_{i} \frac{\tilde{y}_{i-1}}{\tilde{y}_{i+1}} \frac{p_{i}}{p_{i-1}} t_{i-1}+\frac{p_{i}}{\tilde{y}_{i+1}} d_{i}, i=1,2, \ldots, N
$$

Obviously, we must presuppose the $\tilde{y}_{i}$ to be nonzero.

REMARK 3.6. In Nörl und [8, p. 289] order reduction is described with the weights $p_{i}$ all equal to one, thus making the $t_{i}$ the first differences of the $s_{i}$. Note that the actual choice of the $p_{i}$ does not influence the relative stability of the computation of $\left\{t_{i}\right\}_{i=0}^{N}$ (that is with respect to the homogeneous solution of $(3.5)$ ). 
Assume for the moment that $t_{0}$ is known; then $\left\{t_{i}\right\}_{i=1}^{N}$ follows from (3.5). Apparently the desired solution $\left\{x_{i}\right\}_{i=1}^{N+1}$ satisfies the relation $(c f .(3.2)$ and $(3.4))$ :

$$
t_{i}=\left[\frac{x_{i+1}}{\tilde{y}_{i+1}}-\frac{x_{i}}{\tilde{y}_{i}}\right] p_{i}
$$

An approximating sequence, say $\left\{\tilde{x}_{i}^{(N)}\right\}_{i=0}^{N+1}$, can now be found by defining

$$
\tilde{x}_{N+1}^{(N)}=0
$$

and

$$
\tilde{x}_{i}^{(N)}=\frac{\tilde{y}_{i}}{\tilde{y}_{i+1}} \tilde{x}_{i+1}^{(N)}-\frac{t_{i}}{p_{i}} \tilde{y}_{i}, \quad i=N, N-1, \ldots, 0
$$

We still are free to choose $A, B$ and $\left\{p_{i}\right\}_{i=0}^{N}$. If we choose $A=0$, $B=1$ and

$$
p_{i}=-\tilde{y}_{i} \tilde{y}_{i+1}
$$

then we can verify that for all $i, \tilde{y}_{i}=y_{i}$ and, at least for $i \geq 1$, and for all $i, t_{i}=z_{i}$. Moreover we may also identify $t_{0}$ and $z_{0}$, since we may consistently define

$$
t_{0}=\tilde{y}_{1} x_{0}
$$

As a consequence the solution by Olver's algorithm, namely $\left\{x_{i}^{(N)}\right\}_{i=0}^{N+1}$, may be identified with the approximate solution $\left\{\tilde{x}_{i}^{(N)}\right\}_{i=0}^{N+1}$ by the order reduction method in this case.

REMARK 3.12. A generalization of Olver's method for higher order recursions using the reduction method is now obvious. It may however be a hazardous undertaking since in general it is even less clear than it is for the second order case why certain solutions should not have zero's (cf. the 
requirement that, for all $i, y_{i} \neq 0$ ). On the other hand it has been shown in Mattheij [4] that it may lead to a satisfactory algorithm under suitable dominance assumptions.

\section{The Riccati method}

The third method to be used is a discrete analogue of what is often termed invariant imbedding. It is based on using Riccati recursions (as an analogue of Riccati differential equations). We use notation adapted from Meyer [7, p. 99] in order to show the similarity to section 2.

The first step consists of the computation of a solution $\left\{\theta_{i}\right\}_{i=0}^{N}$ to the Riccati recursion

$$
\theta_{i}=\frac{1}{a_{i}+b_{i} \theta_{i-1}}, i=0,1, \ldots, N,
$$

where $\theta_{0}$ is equal to some (given) initial value. These values of the $\theta_{i}$ are then used to compute the solution $\left\{\tau_{i}\right\}_{i=0}^{N}$ to the recursion

$$
\tau_{i}=-b_{i} \theta_{i} \tau_{i-1}-\theta_{i} d_{i}, i=0,1, \ldots, N
$$

where $\tau_{0}$ satisfies

$$
\tau_{0}=c-\theta_{0} x_{1}
$$

(here we must assume that $x_{1}$ has been given unless $\theta_{0}=0$ ). The final step is the "recovery" recursion

$$
x_{i}=\theta_{i} x_{i+1}+\tau_{i}, \quad i=N, N-1, \ldots, \quad .
$$

By choosing $\hat{x}_{N+1}^{(N)}=0$, we can find a solution $\left\{\hat{x}_{i}^{(N)}\right\}_{i=0}^{N+1}$ of (4.4). The similarity to the previous method is most striking. Indeed, just take

$$
\theta_{i}=\frac{\tilde{y}_{i}}{\tilde{y}_{i+1}}, \tau_{i}=-\frac{t_{i} \tilde{y}_{i}}{p_{i}} .
$$

Again we see that we then may identify $\left\{\tilde{x}_{i}^{(N)}\right\}$ with $\left\{\hat{x}_{i}^{(N)}\right\}$ showing Olver's method to be a special case in this instance as well. 
REMARK 4.6. It should be noted that the Riccati method works similarly for higher order recursions ( $c f$. Section 5). In this sense it certainly is a generalization (at least mathematically) of the order reduction method, which may require several successive steps for higher order problems.

\section{Decoupling based on triangularization}

The methods previously described may seem rather ad hoc in the sense that they require the use of substitutions and (scalar) transformations, and so on, in order to obtain some recursions where known initial values and (approximate) terminal values could easily be used. From a numerical point of view, however, the stability is not at all a straightforward and easily deducible matter (in our second order case we know from the similarity as shown in Sections 3 and 4 that there must be stability). Therefore we believe that all these methods become more transparent by considering them as a decoupling algorithm, which separates (relatively) increasing and decreasing modes. To do this consider, more generally, the recursion

$$
x_{i+1}=A_{i} X_{i}+R_{i}, \quad i \geq 0
$$

where the $A_{i}$ are $n \times n$ matrices and the $X_{i}$ are $n$-dimensional solution iterates. Assume we have an initial condition for $\left\{X_{i}\right\}$ given by

$$
M X_{0}=b, M \text { an } n \times n \text { matrix. }
$$

As was shown in Mattheij $[5,6]$ we may transform the $A_{i}$ onto (block) upper triangular form by a sequence of nonsingular $\left\{T_{i}\right\}_{i \geq 0}$, of which $T_{0}$ is given and

$$
A_{i} T_{i}=T_{i+1} U_{i}, U_{i} \text { (block) upper triangular. }
$$

Assume we know the dimension of the dominant solution space, say $k$. Now write

$$
U_{i}:=\left(\begin{array}{cc}
B_{i} & C_{i} \\
0 & E_{i}
\end{array}\right), B_{i} \text { a } k \times k \text { matrix }
$$




$$
\begin{aligned}
& z_{i}:=T_{i}^{-1} X_{i}, s_{i}=T_{i+1}^{-1} R_{i}, \\
& z_{i}:=\left(\begin{array}{c}
z_{i}^{1} \\
z_{i}^{2}
\end{array}\right) . k
\end{aligned}
$$

From (5.1), (5.3) to (5.6) we then obtain the decoupled recursions

$$
z_{i+1}^{2}=E_{i} z_{i}^{2}+S_{i}^{2}, i=0,1, \ldots, N,
$$

$$
z_{i}^{1}=B_{i}^{-1}\left[z_{i+1}^{1}-C_{i} z_{i}^{2}-S_{i}^{1}\right], \quad i=N, N-1, \ldots, 0
$$

(assuming $B_{i}$ is nonsingular).

Now if either $M(c f .(5.2))$ is nonsingular, so we can compute $z_{0}$ explicitly, or $T_{0}$ is chosen in a special way, so that we can find $Z_{0}^{2}$ explicitly, we can use (5.7a). Moreover, by choosing an approximant for $Z_{N+1}^{\perp}$, say $Z_{N+1}^{\perp}(N)=0$, we can compute an approximating sequence $\left\{z_{i}^{1}(N)\right\}_{i=N+1}^{0}$ using $(5.7 \mathrm{~b})$. As an approximant for $x_{i} \quad(0 \leq i \leq N+1)$ we then use

$$
X_{i}(N):=T_{i}\left(\begin{array}{c}
z_{i}^{1}(N) \\
z_{i}^{2}
\end{array}\right) .
$$

For these general recursions (5.1) it has been shown in Mattheij [4,6] that orthogonal matrices are preferable on account of their numerical stability. The analyses in Mattheij $[4,5,6]$ moreover show that the recursions (5.7) in the indicated directions are indeed stable (under very weak conditions, mainly dealing with a proper choice of $T_{0}$ ). In particular this stability is ensured if $M$ has $k$ zero rows, $M$ has rank $(n-k)$ and $T_{0}$ is chosen such that

(5.9) $M T_{0}=\left(\begin{array}{ll}\emptyset & \emptyset \\ \varnothing & H\end{array}\right), H$ an $(n-k)$ th order nonsingular matrix. 
The Riccati method uses special lower triangular matrices $T_{i}$ instead of orthogonal matrices. We describe this first: let $A_{i}$ be partitioned as

$$
A_{i}:=\left(\begin{array}{cc}
A_{i}^{11} & A_{i}^{12} \\
A_{i}^{21} & A_{i}^{22}
\end{array}\right) \downarrow^{\natural k}
$$

and use as matrices $T_{i}$,

$$
T_{i}:=\left(\begin{array}{cc}
I & \emptyset \\
P_{i} & I
\end{array}\right) \uparrow^{\uparrow}
$$

In order to let $U_{i}$ (see (5.3)) have the block upper triangular form as indicated in (5.4), we apparently must require that

(5.12) $-P_{i+1}\left(A_{i}^{12} P_{i}+A_{i}^{11}\right)+A_{i}^{22} P_{i}+A_{i}^{21}=0, i=0,1, \ldots, N-1$.

The decoupled recursion (5.7) then takes the form

(5.13a) $z_{i+1}^{2}=\left[-P_{i+1} A_{i}^{12}+A_{i}^{22}\right] z_{i}^{2}-P_{i+1} R_{i}^{1}+R_{i}^{2}, i=0,1, \ldots, N-1$, (5.13b) $\quad z_{i}^{1}=\left[A_{i}^{12} P_{i}+A_{i}^{11}\right]^{-1}\left[z_{i+1}^{1}-A_{i}^{12} z_{i}^{2}-R_{i}^{1}\right], \quad i=N-1, N-2, \ldots, 0$, and the back transformation (5.8) gives

$$
X_{i}(N)=\left(\begin{array}{c}
z_{i}^{1}(N) \\
P_{i} z_{i}^{1}(N)+z_{i}^{2}
\end{array}\right) .
$$

Now assume the initial condition matrix to be of the form

$$
M=\left[\begin{array}{cc}
\emptyset & \emptyset \\
M^{21} & I
\end{array}\right\}_{\uparrow n-k}
$$

Then we see that the choice

$$
P_{0}=-M^{21}
$$

gives the required form as in (5.9), that is, we can compute $z_{0}^{2}$ explicitly and use (5.13a) and so on. On account of the similarity to the 
algorithm as described in Mattheij [6, Section 5.2], we conclude that the invariant imbedding approach is stable, as long as we can assure that the $P_{i}$ remain bounded, that is, $\left\|T_{i}\right\|,\left\|T_{i}^{-1}\right\|$ is bounded.

REMARK 5.17. If the Riccati method (or any related method) fails because the $P_{i}$ become unbounded, the decoupling method utilizing orthogonal transformations, may still work quite well. For an example of this, see, for example, Mattheij [6, Example 7.1].

We would now like to show that the Riccati method for second order matrix vector recursions, where the $A_{i}$ are companion matrices (that is in fact recursions like (2.1)), boils down to what we described in section 4 . So let us consider

$$
X_{i+1}=\left(\begin{array}{c}
x_{i+2} \\
x_{i+1}
\end{array}\right)=\left(\begin{array}{cc}
a_{i+1} & b_{i+1} \\
1 & 0
\end{array}\right)\left(\begin{array}{c}
x_{i+1} \\
x_{i}
\end{array}\right)+\left(\begin{array}{c}
a_{i+1} \\
0
\end{array}\right), \quad i=0,1, \ldots,
$$

with initial condition

$$
\left(\begin{array}{ll}
0 & 0 \\
0 & 1
\end{array}\right)\left(\begin{array}{l}
x_{1} \\
x_{0}
\end{array}\right)=\left(\begin{array}{l}
0 \\
c
\end{array}\right)
$$

If we apply the preceding method we should choose $P_{0}=0($ cf. (5.16)) and compute $\left\{P_{i}\right\}$ from the relation

$$
-P_{i+1}\left(b_{i+1} P_{i}+a_{i+1}\right)+1=0, i=0,1, \ldots .
$$

After choosing $z_{0}^{2}=c$, we compute $\left\{z_{i}^{2}\right\}_{i=1}^{N}$ from the relation (cf.

$$
z_{i}^{2}=-P_{i} b_{i} Z_{i-1}^{2}-P_{i} d_{i}, \quad i=1,2, \ldots, N \text {. }
$$

For the backward recursion we then find (cf. $(5.12 b))$

$$
\text { (5.22) } \begin{aligned}
x_{i}(N):= & z_{i-1}^{1}(N) \\
& =\left[b_{i} P_{i-1}+a_{i}\right]^{-1}\left[x_{i+1}(N)-b_{i} z_{i-1}^{2}-d_{i}\right], i=N, N-1, \ldots, 0 .
\end{aligned}
$$

From (5.20) and (5.21) we see that (5.22) in fact reads 


$$
x_{i}(N)=P_{i} x_{i+1}(N)+z_{i}^{2}, i=N, N-1, \ldots, 0 .
$$

An interesting aspect of this is that the backward recursion step coincides with the back transformation in a way: the first coordinate transformation is trivial and the second coordinate part of (5.14) is exactly (5.23). of course, we may identify $P_{i}$ and $z_{i}^{2}$ with $\theta_{i}$ and $\tau_{i}$ respectively (see Section 4).

REMARK 5.24. Since we have chosen $P_{0}$ in a special way, we do not need the value of $x_{1}$ in the second order case. However, this choice is not obvious from a stability point of view (it corresponds to a homogeneous solution with initial value 0 ; this solution should dominate $\left\{x_{i}\right\}$ in order to have stability). Therefore it makes sinse to consider a more general Riccati method (that is, with $P_{0} \neq 0$ ) or even another triangularizing sequence $\left\{T_{i}\right\} \quad(c f .(5.3))$ in order to avoid the transformations becoming unbounded. Of course this requires the (approximate) value of $x_{1}$.

Finally, we would like to show how the stability of the preceding algorithms may be investigated using the algebraic decoupling formulation of this section. We assume that $\left\{x_{i}\right\}_{i \geq 0}$ (cf. (2.1)) is dominated by some homogeneous solution $\left\{g_{i}\right\}_{i \geq 0}$ and not dominated by some homogeneous solution $\left\{f_{i}\right\}_{i \geq 0}$, that is, $\lim _{i \rightarrow \infty}\left|x_{i}\right| /\left|g_{i}\right|=0$, there exists $c$ for all $i,\left|f_{i}\right| /\left|x_{i}\right| \leq c$. Moreover we assume that the solution $\left\{x_{i}\right\}_{i \geq 0}$ uniquely exists. For demonstrative reasons we place our derivation in the framework of Mattheij $[5,6]$. Now let $\left\{\Phi_{i}\right\}_{i \geq 0}$ be a fundamental solution of (5.10) with

$$
\Phi_{0}:=T_{0}\left(=\left(\begin{array}{cc}
I & 0 \\
-M^{21} & I
\end{array}\right)\right) \text {. }
$$

Write 
358

R.M.M. Mattie $i j$

$$
\Phi_{i}=\left(\begin{array}{cc}
\phi_{i}^{11} & \phi_{i}^{12} \\
\phi_{i}^{21} & \phi_{i}^{22}
\end{array}\right)=\left(\Phi_{i}^{1} \mid \Phi_{i}^{2}\right) .
$$

It is shown in Mattheij [6, Proposition 5.6] that $\left\{\Phi_{i}\right\}_{i \geq 0}$ then is a consistent fundamental solution, that is, $\left\{\Phi_{i}^{l}\right\}_{i \geq 0}$ is not a dominated solution, and therefore has to be dominant. It is not restrictive therefore to identify $g_{i}$ and $\phi_{i}^{21}$ for all $i$. Obviously there exist constants $\kappa_{1}$ and $\kappa_{2}$ such that $(5.27)$

$$
\phi_{i}^{22}=\kappa_{1} g_{i}+\kappa_{2} f_{i} .
$$

NOTE. $\phi_{i}^{11}=g_{i+1}$ and $\phi_{i}^{12}=\kappa_{1} g_{i+1}+\kappa_{2} f_{i+1}$.

From (5.3), (5.11) and (5.25) we now find

$$
\Phi_{i}=A_{i-1} \cdots A_{0} T_{0}=T_{i} W_{i}:=T_{i} U_{i-1} \cdots U_{0} .
$$

The lower blocks in the relation $T_{i}^{-1} \Phi_{i}=W_{i}$ f note that $W_{i}$ is block upper triangular) give $(5.29 a)$

$$
\begin{aligned}
& -P_{i} g_{i+1}+g_{i}=0, \\
& -P_{i} \phi_{i}^{12}+\phi_{i}^{22}=w_{i}^{22} .
\end{aligned}
$$

Hence from $(5.27),(5.29 a)$ and $(5.29 b)$ we obtain

$$
w_{i}^{22}=\kappa_{2}\left(P_{i} f_{i+1}+f_{i}\right) \text {. }
$$

By construction we see that $w_{i}^{22}=\prod_{0}^{i-1} E_{j} \quad(c f .(5.4))$, where $E_{j}$ is short for $P_{j+1} b_{j+1} \quad(c f .(5.21))$.

Since for the desired solution of (5.21), namely $\left\{z_{i}^{2}\right\}$, there holds $(5.31)$

$$
z_{i-1}^{2}=P_{i} x_{i+1}+x_{i}
$$

https://doi.org/10.1017/S0004972700025867 Published online by Cambridge University Press 
it follows from the dominance assumption that $z_{i-1}^{2}=0\left(w_{i}^{22}\right)=O\left(f_{i}\right)$, that is, (5.21) is stable in forward direction, if only $\left|P_{i}\right|$ is not large for all $i$.

On the other hand, backward recursion via (5.23) is stable since

$$
P_{i}=g_{i+1} / g_{i}
$$

so the homogeneous solutions of (3.23) are dominated by $\left\{x_{i}\right\}$ in backward direction on account of the assumption above.

REMARK 5.33. The main purpose of the above analysis was to indicate how the decoupling actually takes place. As was also shown in Mattheij [5] the crucial point is the factorization $\Phi_{i}=T_{i} W_{i}$; here $T_{i}$ must be a reasonably well conditioned matrix and $W_{i}$ an upper triangular matrix. Note that neither $\Phi_{i}$ nor $W_{i}$ is actually computed (the computation of $\Phi_{i}$ would be unstable of course). However, $W_{i}$ is available, in principle, in factored form as $U_{i-1} \ldots U_{0}$. The $B_{j}, C_{j}$ and $E_{j}$ blocks in the $U_{j}$ are known rather accurately and this justifies the arguments we used to show the stability of the forward recursion (cf. (5.29)-(5.31)).

\section{References}

[1] J.R. Cash, "A note on Olver's algorithm for the solution of secondorder linear difference equations", Math. Comp. 35 (1980), $767-772$.

[2] J.R. Cash, Stable recursions. With applications to the numerical solution of stiff systems (Academic Press [Harcourt Brace Jovanovich ], London, New York, Toronto, 1979).

[3] Daniel W. Lozier, "Numerical solution of linear difference equations" (Report NBSIR 80-1976. National Bureau of Standards, US Department of Commerce, Washington, DC, 1980).

[4] R.M.M. Mattheij, "Estimating and determining solutions of matrix vector recursions" ( $\mathrm{PhD}$ thesis, Utrecht, 1977). 
[5] R.M.M. Mattheij, "Characterizations of dominant and dominated solutions of linear recursions", Numer. Math. 35 (1980), 421-442.

[6] R.M.M. Matthe $i j$, "Stable computation of solutions of unstable linear initial value recursions", BIT 22 (1982), 79-93.

[7] Gunter H. Meyer, Initial value methods for boundary value problems: Theory and application of invariant imbedding (Mathematics in Science and Engineering, 100. Academic Press [Harcourt Brace Jovanovich ], New York and London, 1973).

[8] Niels Erik Nörlund, Vorlesungen über Differenzenrechnung (Chelsea, New York, 1954).

[9] J. Oliver, "The numerical solution of linear recurrence relations", Numer. Math. 11 (1968), 349-360.

[10] F.W.J. Olver, "Numerical solution of second-order linear difference equations", J. Res. Nat. Bur. Standards 71B (1967), 111-129.

[11] F.W.J. Olver and D.J. Sookne, "Note on backward recurrence algorithms", Math. Comp. 26 (1972), 941-947.

[12] J.H. Wilkinson, The algebraic eigenvalue problem (Clarendon Press, Oxford, 1965).

Mathematisch Institut, Katholieke Universiteit, Toernooiveld, Ni jmegen, The Netherlands. 